Relations industrielles

Industrial Relations

\title{
La relation entre l'intensité perçue d'utilisation de la messagerie électronique et la qualité de la relation hiérarchique
}

\section{The relationship between perceived intensity of email use and quality of hierarchical relations \\ La relación entre la intensidad percibida de utilización de la mensajería electrónica y la calidad de la relación jerárquica}

\author{
Géraldine de La Rupelle, Cameron Guthrie et Michel Kalika
}

Volume 70, numéro 1, hiver 2015

URI : https://id.erudit.org/iderudit/1029284ar

DOI : https://doi.org/10.7202/1029284ar

\section{Aller au sommaire du numéro}

\section{Éditeur(s)}

Département des relations industrielles de l’Université Laval

ISSN

0034-379X (imprimé)

1703-8138 (numérique)

Découvrir la revue

Citer cet article

de La Rupelle, G., Guthrie, C. \& Kalika, M. (2015). La relation entre l'intensité perçue d'utilisation de la messagerie électronique et la qualité de la relation hiérarchique. Relations industrielles / Industrial Relations, 70(1), 157-185. https://doi.org/10.7202/1029284ar

\section{Résumé de l'article}

Stress, conflits, surcharge, pression et contrôle, mais aussi gain de temps, motivation, accessibilité... sont autant de mots souvent prononcés à l'évocation de la messagerie électronique, tout particulièrement lorsqu'elle est associée aux relations hiérarchiques.

Bien que les chercheurs et praticiens reconnaissent les effets du média sur la relation hiérarchique, la recherche académique peine à expliquer la nature de leur relation ainsi que les variables explicatives de cette même relation. Visant à répondre à ces questions, la problématique centrale de notre étude consiste à examiner la relation entre l'utilisation de la messagerie électronique et la qualité de la relation hiérarchique en mettant l'accent sur trois variables médiatrices : la reconnaissance, le contrôle et l'accessibilité.

L'étude empirique a été réalisée au sein d'une banque de réseau française. Une étude qualitative exploratoire a contribué à la construction d'échelles de mesure utilisées ensuite dans un dispositif de recherche quantitatif : 478 questionnaires valides ont permis une analyse du modèle par la méthode des équations structurelles.

Les résultats révèlent que l'intensité perçue d’utilisation du média est liée positivement à la qualité de la relation hiérarchique lorsque la relation est considérée isolément des variables médiatrices. En revanche, lorsque nous traitons le modèle dans son ensemble - en introduisant les variables médiatrices de reconnaissance, contrôle et accessibilité - la relation directe entre utilisation de la messagerie électronique et qualité de la relation hiérarchique n'est plus validée. La reconnaissance et le contrôle sont des mécanismes explicatifs de cette relation, tandis que l'accessibilité n'explique que partiellement la relation entre messagerie électronique et qualité de la relation hiérarchique. Outre les apports méthodologiques que procure la construction du modèle et celle des échelles de mesure, la présente recherche vise à fournir des pistes pour une amélioration des pratiques de communication électronique au sein de la dyade supérieur hiérarchique/subordonné.
Tous droits réservés (C Département des relations industrielles de l’Université Laval, 2015
Ce document est protégé par la loi sur le droit d'auteur. L’utilisation des services d'Érudit (y compris la reproduction) est assujettie à sa politique d'utilisation que vous pouvez consulter en ligne.

https://apropos.erudit.org/fr/usagers/politique-dutilisation/ 


\section{La relation entre l'intensité perçue d'utilisation de la messagerie électronique et la qualité de la relation hiérarchique}

\section{Géraldine de La Rupelle, Cameron Guthrie et Michel Kalika}

L'introduction rapide et massive de la messagerie électronique dans les organisations ainsi que son apparente simplicité d'utilisation ont empêché les gestionnaires de se poser a priori les questions autour de son adaptation organisationnelle et culturelle. Si elle est aujourd'hui indéniablement reconnue pour permettre une communication rapide, une forte capacité de réaction ou encore la possibilité de s'affranchir de barrières spatiales et temporelles, elle produit aussi des effets plus inattendus. La messagerie électronique $a$, notamment, entraîné une profonde mutation des relations entre supérieurs hiérarchiques et subordonnés. Dans ce cadre, est-elle alors utilisée pour devenir un instrument de contrôle des collaborateurs, un levier pour mieux motiver les équipes ? Offre-t-elle une opportunité pour se rendre plus accessible ? Et, finalement, l'utilisation du média favorise-t-elle une meilleure qualité de la relation hiérarchique ?

MOTS-CLÉs : Communication électronique, courrier électronique, relation managériale, théorie de l'échange social, relation leader-membre (LMX en anglais).

\section{Introduction}

Le fonctionnement des relations hiérarchiques dans les organisations modernes est en pleine mutation. Ayache et Laroche (2010) proposent quelques pistes d'explication du phénomène : décentralisation des organisations, autonomie et responsabilité grandissante des acteurs ou encore substitution de la communication au commandement. Les managers sont, par ailleurs, confrontés à de nouveaux défis liés à la distorsion des processus de communication due à l'introduction de nouvelles technologies, telles que la messagerie électronique, et doivent apprivoiser les changements induits par la virtualité (Kalika et al., 2007).

Géraldine de la Rupelle, enseignant/chercheur en Systèmes d'information, École supérieure de commerce et de management (ESCEM), campus de Tours et Communauté d'universités et d'établissements (COMUE), Centre-Val de Loire Université, Tours, France (gdelarupelle@escem.fr).

Cameron Guthrie, enseignant/chercheur en Systèmes d'information, Toulouse Business School, Université de Toulouse, France (c.guthrie@tbs-education.fr). 
Une étude des travaux portant sur la relation hiérarchique, d'une part, et sur la communication électronique, d'autre part, permet de mettre en évidence un certain nombre de points communs entre les deux objets de recherche. Si I'on considère que I'un des objectifs de la relation hiérarchique est de réduire l'incertitude et l'ambiguïté (Ayache et Laroche, 2010), il est intéressant de constater que dans les premiers travaux portant sur les médias de communication, Daft et Lengel (1986) posent comme principe de base que si les organisations traitent l'information, c'est également dans l'objectif de réduire l'incertitude et l'ambiguïté. Ainsi existerait-il un objectif commun à la relation hiérarchique et à la communication électronique ? Si l'on considère que la relation hiérarchique contribue à l'efficacité et à la performance d'une organisation (Graen et al., 1982), il est intéressant de constater que de nombreux travaux démontrent la participation des médias de communication à une meilleure productivité de l'organisation (Hildreth, 2001; McMannus et al., 2002 ; Taylor et al., 2008). Ainsi existerait-il une contribution partagée entre la relation hiérarchique et la communication électronique ? Si l'on considère enfin que la relation hiérarchique est un processus qui évolue, se construit et se déploie dans le temps (Graen et Uhl-Bien, 1995; Ayache et Laroche, 2010), il en est de même pour les travaux des structurationnistes en général et ceux d'Orlikowski (1992) en particulier, qui considèrent la technologie comme évoluant dans les dimensions spatiotemporelles. Ainsi, existerait-il une dimension temporelle comparable entre la relation hiérarchique et la communication électronique?

La mise en parallèle d'un objectif commun, d'une contribution partagée et d'une dimension temporelle comparable est intéressante, car elle est le révélateur de l'imbrication de l'outil dans la relation hiérarchique, la preuve que la messagerie vient se mettre au service de la relation hiérarchique; elle nous rappelle également que la construction de la relation passe par la diffusion des informations et par la communication. Mais cette mise en parallèle n'est pas suffisante et peut-être faut-il aujourd'hui s'attarder davantage à démontrer et à comprendre la relation entre l'utilisation de la messagerie électronique et les relations hiérarchiques.

D'un point de vue managérial, les cadres dirigeants de grands groupes se posent aujourd'hui, a posteriori, la question des effets mal pressentis, mal évalués et donc mal maîtrisés du média. Les effets de l'utilisation du média dans le cadre de la relation hiérarchique constituent l'une de leurs préoccupations : réduction des barrières hiérarchiques, implication différente des managers et des subordonnés, effet sur la motivation, augmentation de l'accès aux individus ou encore utilisation de l'outil comme moyen de contrôle.

D'un point de vue théorique, Howell et al. (2005) indiquent le besoin en recherches portant sur la relation entre les managers et leurs collaborateurs, ainsi 
que la fréquence et les modes de communications utilisés. Les travaux de Tahri et Fallery (2010) concluent que, si les recherches portant sur les questions d'utilisation et de satisfaction du média ont fait l'objet de larges vérifications, il serait alors intéressant que les recherches « portent désormais plus d'attention aux liens entre la perception du média et les comportements [...], car les possibilités offertes par le média peuvent être source d'une bonne ou d'une mauvaise appropriation » (p.197).

Dans ce contexte, le questionnement prend tout son sens. S'appuyant sur la théorie de l'échange social (Blau, 1964) et s'intéressant au point de vue des subordonnés, la présente recherche s'attache à vérifier l'hypothèse selon laquelle I'intensité perçue de l'utilisation de la messagerie électronique est en relation avec la qualité de la relation hiérarchique. Nous chercherons ensuite à analyser plus spécifiquement l'effet médiateur de la reconnaissance, du contrôle et de I'accessibilité dans la relation entre l'intensité perçue d'utilisation du média et la qualité de la relation hiérarchique. Les résultats seront susceptibles d'aider les gestionnaires à mettre l'accent sur les variables qui favorisent la qualité de la relation hiérarchique ou à corriger celles qui tendent à la dégrader.

L'article s'organise en quatre parties. La première, adossée aux apports de la littérature, présente le modèle de recherche et formule les hypothèses. La deuxième partie expose la méthodologie de la recherche, décrivant tour à tour le design de la recherche, l'échantillon, la collecte des données et les instruments de mesure. La troisième partie est consacrée à la présentation des résultats tandis que la quatrième éclaire le lecteur sur les apports, limites et perspectives de notre étude.

\section{Revue de littérature, modèle et hypothèses}

Les aspects théoriques préalables à notre travail empirique consistent à s'ancrer dans un courant théorique permettant de mesurer la qualité de la relation hiérarchique du point de vue du subordonné, d'expliquer la relation entre I'intensité d'utilisation de la messagerie électronique et la qualité de cette relation, et, enfin, de justifier le choix de variables explicatives telles que la reconnaissance, le contrôle et l'accessibilité.

\section{Qualité de la relation hiérarchique et utilisation de la messagerie électronique}

Le courant dans lequel s'inscrit notre recherche est celui de l'échange social, défini par Blau (1964) comme "les actes volontaires d'individus motivés par le retour que ces actes sont supposés apporter et qu'ils apportent effectivement de la part des autres »(91-92). Les interactions entre les acteurs sont interdé- 
pendantes et séquentielles, et cette suite d'interactions, grâce à un processus d'auto-renforcement, est censée créer une relation d'échanges de haute qualité (Nasr et al., 2009). La théorie LMX (Leader Member EXchange) prend sa source dans ce courant théorique et permet de mesurer plus spécifiquement la qualité de la relation entre supérieur hiérarchique et subordonné, en étudiant les déterminants suivants : contribution, confiance, affection et respect professionnel. Mesurer la qualité de la relation hiérarchique est central pour notre question de recherche, puisque cela nous permet de déterminer le niveau de satisfaction d'un collaborateur à l'égard des relations qu'il entretient avec son supérieur hiérarchique. Les recherches associées à la LMX démontrent que les supérieurs hiérarchiques développent une relation différente avec chacun des membres de son équipe. Un supérieur hiérarchique peut développer un échange de qualité avec certains subordonnés considérés alors comme plus fiables, plus impliqués et plus loyaux (Dansereau et al., 1975). Ces subordonnés reçoivent plus de temps de la part de leur manager et la communication est plus intense dans le cadre d'une relation de qualité (Niedle, 2012). Avec d'autres subordonnés considérés comme étant hors du groupe, les contacts seront superficiels et plus formels. Afin de favoriser la construction d'un contexte social partagé, les supérieurs hiérarchiques favorisent les échanges d'information (Simard et al., 2005), et développent des comportements adaptés aux échanges électroniques. De la même façon, les subordonnés optent pour des attitudes et comportements aptes à équilibrer la relation (Goh et Wasko, 2012). L'échange interpersonnel qui s'instaure entre le supérieur et ses collaborateurs devient alors déterminant sur le rôle joué par le salarié au sein de son service (Saint-Michel et Wielhorski, 2011). Niedle (2012) démontre que la qualité de la relation entre supérieur et subordonné est liée à la fréquence de la communication.

Certains auteurs considèrent que I'utilisation du média détériore cette qualité de la relation entre un supérieur hiérarchique et ses subordonnés, à l'instar de Hinds et Bailey (2003), de Griffith et al. (2003) et de Boukef (2005) ou, encore, de Van den Hooff (2005) et Taylor et al. (2008) qui évoquent le court-circuit de la hiérarchie. Ces derniers iront même jusqu'à considérer le média comme un instrument de harcèlement dans les cas extrêmes. Un second courant considère, au contraire, la communication électronique comme un facteur d'amélioration de la communication organisationnelle : parmi eux, McManus et al. (2002) estiment que le média facilite et améliore la communication directe; Straus et Mc Grath (1994) affirment que le média favorise le travail collaboratif; tandis que pour Huang (2002), le média joue un rôle de réseau social, dans la mesure où son utilisation permet d'intensifier les relations interpersonnelles entre supérieurs hiérarchiques et subordonnés. L'opposition entre ces deux courants est, d'ailleurs, mise en évidence dans un certain nombre de recherches qui parlent alors d'effets contradictoires du média (Bia Figueiredo et Kalika, 2010; de la Rupelle, 2010). 
Précisons, enfin, que nous envisageons l'usage de la messagerie électronique sous l'angle de son degré d'utilisation par les utilisateurs, ce que nous appellerons intensité. En 2010, Tahri et Fallery procèdent à une méta-analyse des travaux portant sur la messagerie électronique et relèvent le principe selon lequel «I'utilisation correspond généralement à la mesure du volume ». Certains auteurs mesurent le nombre d'envois et de réceptions de messages (Tahri et Fallery, 2009), d'autres le nombre de consultations du logiciel de messagerie (Lemarié et Wageman, 2000; Mano et Mesch, 2010). Rares sont les auteurs prenant en compte la perception de I'utilisateur au regard de l'intensité d'utilisation du média; à l'instar de Rosanvallon (2011), et selon les recommandations de Tahri et Fallery (2009), notre choix se porte sur la mesure de cette perception plutôt que des volumes afin de prendre en compte le ressenti de l'utilisateur.

L'expression la plus simple de notre problématique centrale repose sur la relation directe entre l'intensité d'utilisation de la messagerie électronique et la qualité de la relation hiérarchique : elle traduit le simple constat que l'intensité d'utilisation du média, perçue par le subordonné, peut avoir des effets sur la qualité de la relation hiérarchique. Le manque de consensus observé dans la littérature nous interdit de prédire si cette relation sera positive ou négative; nous pouvons, dès lors, formuler I'hypothèse sans donner le sens de cette relation entre les deux variables:

H1 : I'intensité perçue d'utilisation de la messagerie électronique est en relation avec la qualité de la relation hiérarchique.

Les résultats d'une étude qualitative exploratoire (de la Rupelle et Kalika, 2009) montrent que l'intensité perçue d'utilisation du média dans la relation hiérarchique est liée non seulement à la qualité de la relation elle-même, mais qu'elle a également un lien avec la structure que représente la dyade supérieur hiérarchique/subordonné en matière de reconnaissance, de contrôle et d'accessibilité. Ce sont ces trois variables que nous retenons comme étant des variables explicatives, et donc médiatrices, dans notre modèle de recherche. Nous validerons cette position dans la section suivante par le recensement d'un grand nombre de recherches allant dans ce sens.

\section{Reconnaissance, contrôle et accessibilité}

Sproull et Kiesler (1991) observent les effets produits par I'utilisation de la messagerie électronique et ils distinguent les effets produits sur les individus (stress, surcharge, sentiment d'urgence, pression...) des effets produits sur les structures sociales. Ces derniers concernent notamment les effets sur les relations et les interactions au sein des structures. La reconnaissance, le contrôle et l'accessibilité sont des variables qui s'inscrivent dans ce second type d'effets et qui vont permettre de mieux expliquer la relation entre l'utilisation du média et la qualité de la relation hiérarchique. 


\section{Reconnaissance}

El Akremi (2009) nous rappelle que " la reconnaissance est au centre des préoccupations des salariés. En développant envers la personne du travailleur des signes de reconnaissance authentique, [...] la confiance est alors renforcée ». Dans le cadre de la théorie de l'échange social, Wils et Labelle (2005) avancent que les employés adaptent leur comportement en échange de récompenses reçues; les auteurs précisent que les récompenses peuvent se concrétiser, entre autres, par des marques de reconnaissance que les employés reçoivent de la part de leur supérieur hiérarchique. Simard et al. (2005) vont dans le même sens, en indiquant que la reconnaissance peut se manifester par des félicitations ou une reconnaissance symbolique de la part du supérieur immédiat. El Akremi (2009) avance les diverses formes que peut prendre la reconnaissance : respect, sentiment d'utilité, esprit de solidarité ou encore remerciements.

En 2000, Lemarié et Wageman analysent des situations de communication par messagerie chez les cadres dans leur rôle de leader; ils notent que le média permet de féliciter un subordonné et lui assurer un retour immédiat sur une tâche accomplie. Boukef-Charki et Charki (2009) démontrent que la messagerie électronique permet une meilleure implication des subordonnés par leur responsabilisation et par leur plus grande autonomie. Cette plus grande autonomie dans l'exécution des tâches grâce à la technologie est reprise par Morin (2000). Hodson (2001) suggère que la technologie permet au salarié de progresser dans ses compétences intellectuelles en augmentant, par exemple, ses capacités en résolution de problème(34). Pour Simard et al. (2005), « les pratiques de partage d'information [...] sont considérées comme la base de l'implication et de la mobilisation des salariés » (299). Complétant les recherches précédentes, notre étude exploratoire a mis en évidence le fait que la messagerie peut être un vecteur de reconnaissance sous trois formes différentes : a) féliciter, remercier ou encourager un collaborateur sur une tâche réalisée ou à venir; b) partager l'information et donner ainsi plus d'autonomie à un collaborateur; c) répondre rapidement aux courriels d'un collaborateur et lui donner ainsi une marque d'intérêt et d'estime.

Sur la base de ces recherches, nous sommes en mesure d'avancer l'hypothèse générale selon laquelle :

H2 : la reconnaissancemédiatise positivementla relation entrel'intensité perçue d'utilisation de la messagerie électronique et la qualité de la relation hiérarchique.

\section{Contrôle}

Dès 1975, Foucault considère que les TIC sont à l'origine d'un contrôle panoptique basé sur le principe de la visibilité. II est vrai que I'usage des TIC procure une visibilité accrue à travers les informations reçues; à ce titre, elle est considérée 
par Craipeau et Seys en 2005 comme un « prolongement du regard ». Morin (2000) nous rappelle que la technologie permet, incite ou force les organisations à modifier leurs processus de contrôle et pose dès lors la question d'une libération ou d'un asservissement du travailleur. Lalle (1999) définit les nouvelles formes de contrôle liées à la mise en œuvre des technologies de l'information; elle met alors en évidence le développement d'un contrôle intégré aux processus de travail par l'intermédiaire des outils informatiques et le développement d'un contrôle externe par les flux, les rythmes de travail ou, encore, I'urgence des tâches à réaliser. Hodson (2001) va dans le même sens, en indiquant que la technologie peut conduire à une surveillance accrue du salarié, mais nuance sa position en ajoutant qu'elle peut également donner du pouvoir à ce dernier grâce à sa capacité à comprendre et contrôler la technologie (p.265). Leclercq (2008, p.38) précise que « la transmission de l'information s'avère fondamentale, tant de façon descendante afin de commander et maîtriser les actions des subordonnés, que de façon ascendante, afin de vérifier et mesurer les performances de ces derniers ». Ainsi, la technologie offre-t-elle " des modalités de contrôle inédites et performantes qui s'ajoutent ou se substituent à celles qui existent déjà » (Greenan et al., 2012 : 39).

En 1991, Sproull et Kiesler détectent une réduction du contrôle vertical du fait de l'utilisation de la messagerie électronique, mais ils seront peu suivis dans la littérature. Le média est plutôt identifié comme étant l'occasion d'un renforcement du contrôle (Nolan, 2003; Taylor et al., 2008; Boukef-Charki et Charki, 2009). Selon d'autres auteurs, il permet une plus grande traçabilité et un autocontrôle a posteriori des tâches accomplies ou non (Vaujany, 2001 ; Boukef, 2005), et il permet également de contrôler les procédures de travail pour en favoriser l'adoption et l'uniformisation. Enfin, il est considéré comme un outil de surveillance et un moyen de contrôler la performance (Taylor et al., 2008).

Par ailleurs, les travaux d'Alie (2007), s'inscrivant dans le courant de l'échange social, attestent que dans le cadre d'un échange, « la confiance ne semble pas vraiment pouvoir émerger lorsque l'employé sent qu'on cherche à le contrôler » (20). Les travaux de Giddens (1989), et plus largement ceux des structurationnistes, affirment, quant à eux, que les subordonnés n'acceptent pas de façon passive le contrôle du manager. Foucault (1975) évoque même diverses possibilités de résistances et de rébellions. Cela nous amène à proposer que le contrôle puisse médiatiser négativement la qualité de la relation hiérarchique.

L'ensemble de ces recherches nous permet donc d'avancer l'hypothèse suivante :

H3 : le contrôle médiatise négativement la relation entre l'intensité perçue d'utilisation de la messagerie électronique et la qualité de la relation hiérarchique. 


\section{Accessibilité}

Selon Morin (2000), « la présence/disponibilité réelle et intégrale du salarié en un lieu précis [...] n'est plus toujours nécessaire grâce aux nouvelles technologies ». II insiste sur le fait que cette « présence virtuelle » est applicable à tout le personnel et nous conduit à nous interroger sur les conséquences de cette « télésubordination ». La messagerie électronique influence la fréquence ou le volume des relations : Ducheneaut et Bellotti (2001) soulignent qu'une proportion croissante des échanges intra-organisationnels transite par le média; Van den Hooff relève, en 2005, un accroissement de la communication organisationnelle et une amélioration de la connectivité; McMannus et al. (2002) soulignent également l'augmentation de l'accès aux individus. En 2005, Assadi et Denis définissent le média comme un opérateur de flexibilité communicationnelle, utilisant tour à tour les termes d'accessibilité, de joignabilité et de visibilité. La sensation de la présence de l'autre, alors même qu'il est physiquement absent, est accentuée par l'accessibilité et la disponibilité procurées par le média (Biocca et Harms, 2002). Les personnes interviewées lors de notre phase qualitative exploratoire soulignent le fait que la messagerie leur permet de mieux gérer leur propre absence physique ou celle de leur supérieur hiérarchique. Loin des contraintes spatiales ou temporelles, le média permet à chacun d'améliorer sa propre accessibilité ou celle de son interlocuteur. Il y a de nombreuses références dans la littérature sur l'accessibilité, la joignabilité et la disponibilité accrues grâce à l'utilisation du média, entre autres dans le cadre de la relation hiérarchique (Huang, 2002; de la Rupelle, 2010; Bachelet et Moscarola, 2001). Mais il n'existe pas de consensus sur le lien entre cette accessibilité et la qualité de la relation hiérarchique : pour certains auteurs, elle s'avère un gage d'une amélioration de la relation, tandis que pour d'autres, elle contribue un peu plus au phénomène de surcharge communicationnelle. Nous choisissons donc de ne pas donner de sens à la relation de l'hypothèse 4.

H4: I'accessibilité médiatise la relation entre l'intensité perçue d'utilisation de la messagerie électronique et la qualité de la relation hiérarchique.

L'ensemble des variables et hypothèses est représenté sur la figure 1.

\section{Méthodologie de la recherche}

La démarche de notre recherche s'inscrit dans une logique de combinaison d'une approche qualitative exploratoire et d'une approche quantitative confirmatoire (Thiétart, 2007). La phase qualitative de nos travaux nous a permis de cerner la question de recherche auprès d'un nombre restreint d'utilisateurs de la messagerie électronique. L'analyse de nos données qualitatives (de la Rupelle et Kalika, 2009) nous a amenés à mettre en évidence et à justifier le choix des variables de notre modèle. 


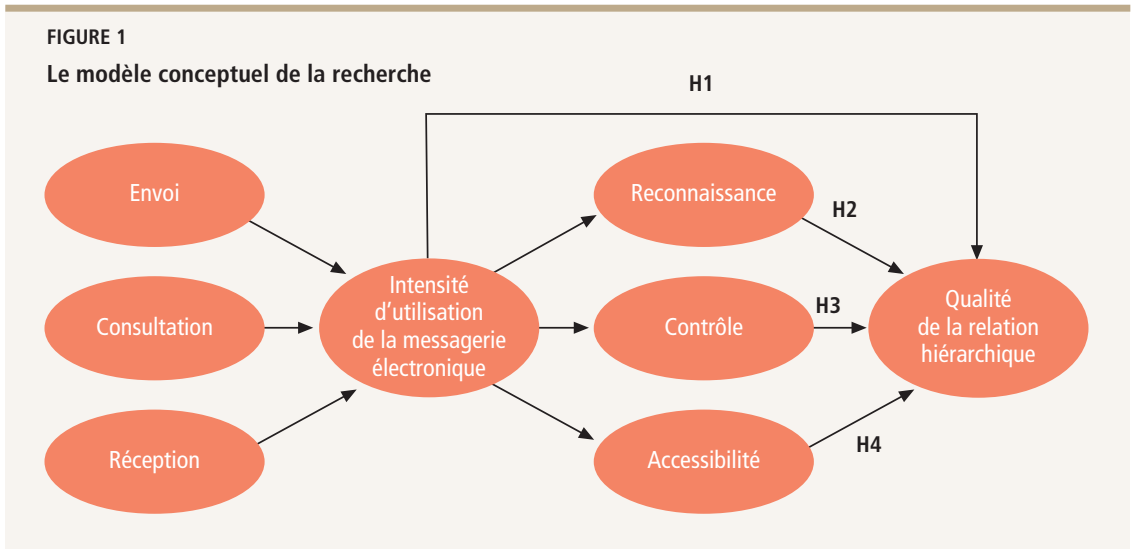

La phase quantitative de nos travaux cherche à généraliser nos conclusions en collectant des données auprès d'un échantillon représentatif de ces mêmes utilisateurs. L'objectif de cette phase de notre recherche est de mesurer les variables du modèle et de tester les hypothèses de recherche qui en découlent (Creswell, 2003), cela par le biais de tests empiriques.

Le terrain retenu est un grand groupe bancaire français. Le choix d'une seule entreprise est justifié par la volonté d'isoler le contexte organisationnel comme facteur modérateur de nos hypothèses de recherche. Le choix de ce terrain s'appuie sur trois éléments. Le niveau d'adoption de la technologie tout d'abord : la messagerie électronique au sein du groupe est considérée comme un média de communication interpersonnelle, au même titre que les médias plus classiques tels que le téléphone, les rendez-vous ou encore les réunions. Les effets structurels ensuite : une double structure hiérarchique - aplatie dans les agences bancaires et, au contraire, verticalisée vers les directions départementales, régionales et nationales - constitue un terrain très riche d'observation de la relation perçue entre l'utilisation du média et la qualité de la relation hiérarchique. La préoccupation des dirigeants du groupe bancaire enfin : le sujet de notre recherche correspond à un questionnement, voire une inquiétude, de la part de la direction du groupe qui s'interroge sur les conséquences du média sur le climat social au sein du groupe. C'est d'ailleurs en concertation avec les partenaires sociaux (représentants du personnel et syndicats) que la direction du groupe nous a ouvert l'accès au terrain.

\section{Échantillon}

La population étudiée est l'ensemble des salariés de la banque de réseau. Un échantillon de 2684 salariés a été constitué sur la base de la représentativité des critères suivants : sexe, âge, niveau hiérarchique et fonction. Nous avons répertorié 305 individus pour lesquels l'adresse courriel n'était pas valide, ce qui a réduit à 2329 le nombre d'individus ayant effectivement reçu le questionnaire. 
Le questionnaire construit pour notre étude comporte 33 items et a été envoyé par messagerie électronique. La possibilité était donnée au répondant de remplir le questionnaire électroniquement et de le retourner par messagerie, ce qui n'altère en rien la qualité des réponses (Cerdin et Peretti, 2001); en revanche, dans la mesure où l'on note une plus grande inquiétude quant au respect de l'anonymat que pour les méthodes de recueil traditionnel (Cerdin et Peretti, 2001), nous avons laissé aux répondants la possibilité de retourner leur questionnaire par la voie postale classique. Le pourcentage des retours par courriel a été de $68 \%$ et celui des retours par voie postale de $32 \%$.

Sur les 2329 salariés, 483 nous ont retourné le questionnaire, soit un taux de retour de $21 \%$. Cinq questionnaires ont été exclus en raison d'un nombre trop important de réponses manquantes, portant à 478 le nombre de questionnaires exploitables. Le tableau 1 présente les principales caractéristiques de l'échantillon.

\begin{tabular}{|c|c|c|c|c|c|}
\hline \multicolumn{6}{|c|}{ Caractéristiques de l'échantillon } \\
\hline Age & Sexe & $\begin{array}{c}\text { Ancienneté } \\
\text { banque }\end{array}$ & Ancienneté & Encadrement & Niveau étude \\
\hline$x=43,6$ ans & $H: 57 \%$ & $x=21,9$ ans & $x=19$ ans & Non cadre : $21 \%$ & Bac ou - : $26 \%$ \\
\hline \multirow[t]{3}{*}{$s=10,7$} & $F: 43 \%$ & $s=12,2$ & $s=13,1$ & Cadre : $74 \%$ & $\mathrm{Bac}+1$ ou $2: 24 \%$ \\
\hline & & & & Cadre dirigeant : $5 \%$ & $\mathrm{Bac}+3$ ou $4: 11 \%$ \\
\hline & & & & & $\mathrm{Bac}+5 \mathrm{ou}+: 39 \%$ \\
\hline
\end{tabular}

\section{Instruments de mesure}

Toutes les variables sont mesurées à l'aide d'échelles de Likert à sept points (de 1 = pas du tout d'accord, à 7 = tout à fait d'accord). Une variable est mesurée à l'aide d'une échelle issue de la littérature : la qualité de la relation hiérarchique. L'intensité perçue d'utilisation de la messagerie électronique, la reconnaissance, le contrôle et l'accessibilité sont mesurés par des échelles créées pour l'étude.

La construction des quatre nouvelles échelles a suivi une démarche en cinq étapes. Dans un premier temps, nous nous sommes appuyés sur une revue de littérature pour construire un guide d'entretien. Nous avons, ensuite, mené des entretiens semi-directifs avec dix individus en tant que supérieurs hiérarchiques et dix autres en tant que subordonnés travaillant dans deux organisations différentes. Ces deux premières phases nous ont permis de voir dans quelle mesure les descripteurs pertinents du sujet de recherche déjà identifiés dans la littérature étaient présents dans le terrain étudié et aussi d'identifier des descripteurs pertinents qui n'avaient pas encore été mentionnés par des chercheurs (Romelaer, 2005). 
Dans un troisième temps, les données ont été codées, puis exploitées à l'aide du logiciel d'analyse de données qualitatives Nvivo afin d'identifier plusieurs items décrivant chacune des variables. Les échelles ont, ensuite, fait l'objet de deux prétests auprès d'un échantillon de 27 individus.

Une fois les énoncés des instruments de mesure construits, nous avons procédé à un certain nombre d'allers-retours entre la collecte des données, la purification des échelles et la mesure de leur validité et fiabilité selon le paradigme de Churchill (1979). II s'agit d'intégrer les connaissances de la littérature afin de créer ou améliorer un instrument de mesure lui-même, qui sera ensuite utilisé dans un questionnaire. Nous nous sommes appuyés sur les recherches antérieures en Systèmes d'information et sur notre phase qualitative pour libeller les items des instruments de mesure. L'objectif était de construire une échelle comprenant idéalement entre 4 à 6 items (Hinkin, 2005).

Enfin, pour chaque variable, nous avons entrepris une analyse factorielle exploratoire afin d'épurer les échelles. Tous les items dont la qualité de représentation est jugée comme étant insuffisante, c'est-à-dire inférieure à 0,6 ont été supprimés. Au préalable, nous n'avions validé que les données s'y prêtaient par un test de sphéricité de Bartlett et le calcul de l'indice Kaiser, Meyer et Olkin. Nous avons ensuite vérifié la fiabilité de nos échelles à l'aide de l'alpha de Cronbach.

\section{L'intensité perçue d'utilisation de la messagerie électronique}

L'intensité perçue d'utilisation de la messagerie électronique est formée de trois dimensions : I'envoi, la réception et la consultation. Selon Tahri et Fallery (2009), I'utilisation du média peut être mesurée par le nombre de messages reçus ou envoyés; à ces deux actions nous avons ajouté la consultation, également présente dans la littérature (Lemarié et Wageman, 2000; Mano et Mesch, 2010), et fortement déterminante de la façon dont l'individu utilise le média.

Nous avons construit une échelle pour mesurer chacune des trois dimensions, toujours sous l'angle de la perception des utilisateurs. Quatre questions portent sur l'envoi du courrier électronique, quatre questions concernent la consultation de messagerie et cinq questions abordent la réception du courrier. Les statistiques de cohérence interne (alpha de Cronbach) sont respectivement 0,94, 0,82 et 0,92. Elles sont toutes satisfaisantes, car elles dépassent le seuil de 0,70 proposé par Nunally (1978).

Suivant les recommandations de Tahri et Fallery (2010), les échelles sont volontairement construites sur la base de la perception des utilisateurs et non sur une mesure effective des messages reçus, envoyés ou du nombre de consultations du logiciel de messagerie. Ce choix permet de prendre en compte le ressenti de I'utilisateur dans son rapport avec l'outil. 


\section{La reconnaissance}

Nous avons développé une échelle pour mesurer la reconnaissance adaptée au contexte des échanges par messagerie électronique; elle comporte cinq items. L'alpha de Cronbach est de 0,86.

\section{Le contrôle}

Nous avons développé une échelle pour mesurer le contrôle dans un contexte d'échanges par messagerie électronique. II s'agit d'une échelle composée de quatre items. L'alpha de Cronbach de 0,81 confirme la fiabilité de cet instrument.

\section{L'accessibilité}

Une échelle de quatre items mesure l'accessibilité dans un contexte d'échange par courrier électronique. Nous avons développé cette échelle pour les besoins de l'étude. L'alpha de Cronbach de 0,78 est d'un niveau satisfaisant.

\section{La qualité de la relation hiérarchique}

Pour la qualité de la relation hiérarchique, I'échelle utilisée est la Leader-Member Exchange, LMX (Graen et Cashman, 1975; Linden et Graen, 1980; Graen et Uhl-Bien, 1995). Elle part du principe que les supérieurs hiérarchiques et leurs subordonnés sont capables de développer des « relations matures » — ils parlent même de partenariat — et de tirer parti des nombreux bénéfices induits par cette relation. L'échelle LMX permet d'évaluer la qualité de la relation hiérarchique en étudiant les déterminants suivants : contribution, confiance, affection et respect professionnel. Dans sa version initiale, il s'agit d'une échelle à 4 points comportant 7 items. À l'instar de Klein et Kim (1998), de nombreux auteurs I'ont modifiée, passant de 4 à 5 points. Suivant leur logique et afin d'obtenir une mesure plus fine des perceptions, nous avons opté pour une échelle en 7 points.

Nous avons traduit l'instrument en français, selon une technique de rétro traduction (Forgues, 1995). II s'agit d'une procédure largement répandue en sciences sociales pour atteindre l'équivalence lexicale qui consiste à demander à deux traducteurs natifs de la langue anglaise de traduire l'instrument en français et, ensuite, à deux traducteurs natifs de la langue française de retraduire cette version. Nous avons complété cette première série de traductions par une traduction parallèle (Malhotra et al., 2007). En s'appuyant sur l'ensemble des documents, les items traduits de façon identique ont été conservés, les autres ont fait l'objet de débats entre les traducteurs qui ont discuté des différentes versions possibles jusqu'à l'obtention d'un consensus. L'alpha de Cronbach de 0,92 confirme la cohérence interne de cet instrument de mesure.

Le tableau 2 présente les résultats des tests pour chacune des variables. 


\begin{tabular}{|c|c|c|c|c|}
\hline \multicolumn{4}{|c|}{$\begin{array}{l}\text { TABLEAU } 2 \\
\text { Résultats des tests d'échelles de mesure }\end{array}$} & \multirow[b]{2}{*}{$\begin{array}{l}\text { Alpha de } \\
\text { Chronbach }\end{array}$} \\
\hline Variable & Source & $\begin{array}{c}\text { Test de sphéricité } \\
\text { de Bartlett }\end{array}$ & Indice KMO & \\
\hline Envoi & Créée & $X^{2}=1809 ; p<0,0001$ & 0,90 & 0,94 \\
\hline Consultation & Créée & $X^{2}=770 ; \quad p<0,0001$ & 0,78 & 0,82 \\
\hline Réception & Créée & $X^{2}=1791 ; p<0,0001$ & 0,90 & 0,92 \\
\hline Reconnaissance & Créée & $X^{2}=1489 ; p<0,0001$ & 0,84 & 0,86 \\
\hline Contrôle & Créée & $X^{2}=1317 ; p<0,0001$ & 0,84 & 0,81 \\
\hline Accessibilité & Créée & $X^{2}=1636 ; p<0,0001$ & 0,60 & 0,78 \\
\hline $\begin{array}{l}\text { Qualité relation } \\
\text { hiérarchique }\end{array}$ & $\begin{array}{l}\text { LMX (Graen et Cashman, } \\
1975 \text {; Liden et Graen, 1980) }\end{array}$ & - & - & 0,92 \\
\hline
\end{tabular}

\section{Résultats}

Afin de tester notre modèle de recherche, nous avons adopté une méthodologie d'équations structurelles, estimées par une approche de moindres carrés partiels (" partial least squares », PLS), en employant le logiciel SmartPLS (Ringle et al., 2005). L'approche PLS applique la régression en moindres carrés partiels pour l'estimation des modèles d'équations structurelles. Elle est particulièrement adaptée au cas où le modèle structurel comporte à la fois des construits formatifs et réflexifs. Dans notre modèle, l'intensité perçue de la messagerie électronique est une variable formative de second ordre, tandis que les autres variables sont réflexives.

Nous présentons les résultats en deux temps : d'abord le modèle de mesure puis le modèle structurel.

\section{Le modèle de mesure}

Nos premières analyses ont porté sur la qualité des mesures. Nous avons, notamment, vérifié la cohérence interne ainsi que la validité convergente et discriminante des cinq instruments. Comme indiqué précédemment, toutes les statistiques de cohérence interne dépassent le seuil d'acceptation de 0,70 (Nunnally, 1978). Les résultats des tests de validité sont présentés en tableaux 3 et 4 . La variance moyenne extraite (" average variance extracted ", AVE) pour chaque variable est supérieure au minimum acceptable de 0,5 (Fornell et Larcker, 1981), ce qui indique la validité convergente des items.

Nous avons, ensuite, testé la validité discriminante des mesures du modèle. La racine carrée de l'AVE est supérieure à sa corrélation avec chacun des autres 
TABLEAU 3

Validité convergente des mesures

\begin{tabular}{lc} 
Variable & Variance moyenne extraite (AVE) \\
Envoi & 0,84 \\
\hline Réception & 0,76 \\
\hline Consultation & 0,65 \\
\hline Reconnaissance & 0,63 \\
\hline Contrôle & 0,64 \\
\hline Accessibilité & 0,58 \\
\hline LMX & 0,69
\end{tabular}

\section{TABLEAU 4}

Validité discriminante et inter-corrélations des variables manifestes

\begin{tabular}{|c|c|c|c|c|c|c|c|c|}
\hline $\begin{array}{l}\text { Va } \\
1\end{array}$ & $\begin{array}{l}\text { ariables latentes } \\
\text { Envoi }\end{array}$ & $\begin{array}{c}1 \\
0,84\end{array}$ & 2 & 3 & 4 & 5 & 6 & 7 \\
\hline 2 & Consultation & 0,37 & 0,65 & & & & & \\
\hline 3 & Réception & 0,63 & 0,31 & 0,76 & & & & \\
\hline 4 & Reconnaissance & 0,18 & 0,22 & 0,14 & 0,63 & & & \\
\hline 5 & Contrôle & 0,19 & 0,24 & 0,17 & 0,33 & 0,64 & & \\
\hline 6 & Accessibilité & 0,05 & 0,07 & 0,03 & 0,28 & 0,10 & 0,58 & \\
\hline 7 & LMX & 0,07 & 0,06 & 0,09 & 0,25 & $-0,12$ & 0,24 & 0,69 \\
\hline
\end{tabular}

construits du modèle, ce qui confirme la validité discriminante de nos échelles de mesure (tableau 4, AVE en gras).

Les résultats pour la validité convergente des variables sont présentés en tableau 5. Nous notons que : les corrélations entre les mesures et leurs facteurs varient de 0,61 à 0,94 ; elles sont plus élevées que les intercorrélations avec d'autres construits. Par ailleurs, la corrélation de chaque mesure avec son facteur respectif est très significative $(p<0,0001)$. Les ratios critiques de chaque mesure sont tous supérieurs à 5,86 .

\section{Le modèle structurel}

Le modèle structurel est présenté en figure 2. La plupart des chemins structurels sont très significatifs $(p<0,001)$ et dans la direction attendue. Une seule relation n'est pas significative, celle entre l'intensité perçue d'utilisation de la 
TABLEAU 5

Les contributions factorielles des variables manifestes

Envoie Consultation Réception Reconnaissance Contrôle Accessibilité LMX

\begin{tabular}{|c|c|c|c|c|c|c|c|}
\hline ENV1 & 0,94 & 0,35 & 0,55 & 0,17 & 0,16 & 0,02 & 0,06 \\
\hline ENV2 & 0,94 & 0,37 & 0,54 & 0,20 & 0,18 & 0,06 & 0,08 \\
\hline ENV3 & 0,93 & 0,31 & 0,53 & 0,15 & 0,18 & 0,04 & 0,01 \\
\hline ENV4 & 0,87 & 0,34 & 0,70 & 0,14 & 0,18 & 0,07 & 0,10 \\
\hline CON1 & 0,24 & 0,82 & 0,17 & 0,14 & 0,15 & $-0,02$ & 0,00 \\
\hline CON2 & 0,26 & 0,84 & 0,19 & 0,15 & 0,20 & 0,06 & 0,07 \\
\hline CON3 & 0,42 & 0,84 & 0,36 & 0,21 & 0,22 & 0,10 & 0,05 \\
\hline CON4 & 0,24 & 0,72 & 0,24 & 0,20 & 0,20 & 0,07 & 0,06 \\
\hline REC1 & 0,55 & 0,22 & 0,88 & 0,14 & 0,14 & 0,55 & 0,22 \\
\hline $\mathrm{REC2}$ & 0,52 & 0,31 & 0,88 & 0,13 & 0,12 & 0,52 & 0,31 \\
\hline REC3 & 0,61 & 0,30 & 0,92 & 0,11 & 0,16 & 0,61 & 0,30 \\
\hline REC4 & 0,45 & 0,22 & 0,75 & 0,08 & 0,20 & 0,45 & 0,22 \\
\hline REC5 & 0,62 & 0,28 & 0,91 & 0,13 & 0,15 & 0,62 & 0,28 \\
\hline мот1 & 0,20 & 0,24 & 0,15 & 0,71 & 0,23 & 0,16 & 0,10 \\
\hline Мот2 & 0,14 & 0,26 & 0,09 & 0,77 & 0,27 & 0,19 & 0,09 \\
\hline Мот3 & 0,15 & 0,10 & 0,10 & 0,80 & 0,21 & 0,23 & 0,31 \\
\hline Мот4 & 0,10 & 0,17 & 0,10 & 0,86 & 0,30 & 0,28 & 0,26 \\
\hline Мот5 & 0,13 & 0,16 & 0,11 & 0,83 & 0,30 & 0,22 & 0,15 \\
\hline CRL1 & 0,10 & 0,17 & 0,07 & 0,28 & 0,61 & 0,07 & 0,02 \\
\hline CRL2 & 0,16 & 0,22 & 0,13 & 0,30 & 0,86 & 0,12 & $-0,08$ \\
\hline CRL3 & 0,17 & 0,17 & 0,18 & 0,28 & 0,89 & 0,08 & $-0,12$ \\
\hline CRL4 & 0,16 & 0,21 & 0,15 & 0,23 & 0,82 & 0,05 & $-0,15$ \\
\hline $\mathrm{ACC} 1$ & 0,01 & 0,04 & $-0,02$ & 0,27 & 0,11 & 0,88 & 0,26 \\
\hline ACC2 & 0,07 & 0,09 & 0,02 & 0,23 & 0,08 & 0,86 & 0,21 \\
\hline $\mathrm{ACC} 3$ & 0,04 & 0,04 & 0,07 & 0,17 & 0,04 & 0,66 & 0,12 \\
\hline ACC4 & 0,04 & 0,07 & 0,07 & 0,16 & 0,06 & 0,63 & 0,08 \\
\hline LMX1 & 0,09 & 0,02 & 0,06 & 0,17 & $-0,10$ & 0,20 & 0,79 \\
\hline LMX2 & 0,05 & 0,05 & 0,06 & 0,22 & $-0,09$ & 0,22 & 0,87 \\
\hline LMX3 & 0,11 & 0,07 & 0,08 & 0,17 & $-0,15$ & 0,22 & 0,87 \\
\hline LMX4 & 0,09 & 0,08 & 0,12 & 0,23 & $-0,05$ & 0,17 & 0,76 \\
\hline LMX5 & 0,02 & 0,04 & 0,04 & 0,21 & $-0,06$ & 0,23 & 0,82 \\
\hline LMX6 & 0,00 & $-0,01$ & 0,08 & 0,21 & $-0,13$ & 0,18 & 0,80 \\
\hline LMX7 & 0,05 & 0,09 & 0,08 & 0,24 & $-0,10$ & 0,19 & 0,89 \\
\hline
\end{tabular}




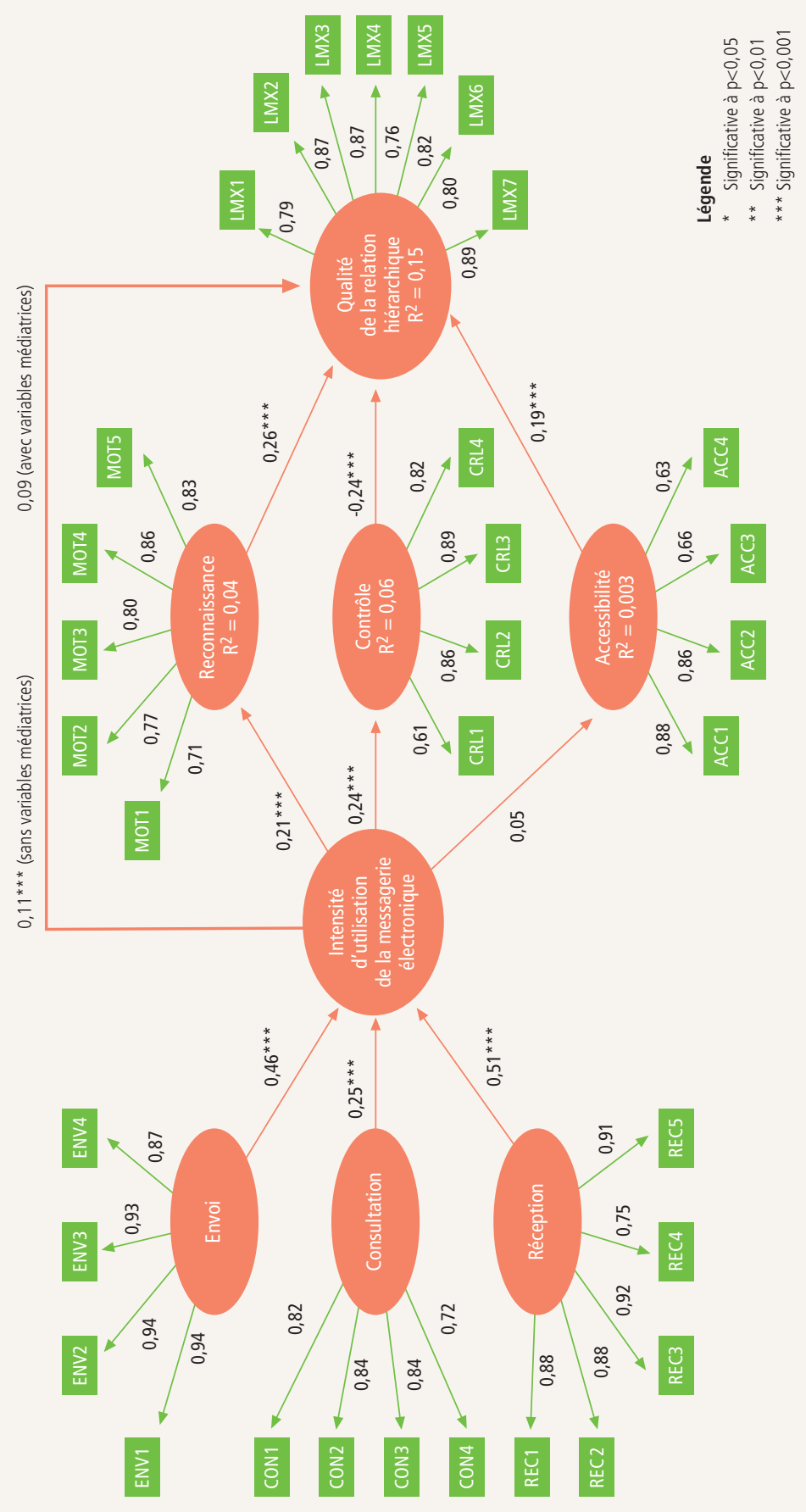


messagerie électronique et l'accessibilité $(\beta=0,05, p=0,25)$. Par ailleurs, I'effet direct entre l'intensité perçue d'utilisation de la messagerie électronique et la qualité de la relation hiérarchique $(\beta=0,11, p<0,001)$ n'est plus significative après I'introduction des variables médiatrices $(\beta=0,09, p=0,12)$ (voir sections suivantes).

\section{Effet direct de l'intensité perçue d'utilisation du média sur la relation hiérarchique}

Les résultats de la figure 2 montrent que la relation entre l'intensité perçue d'utilisation de la messagerie électronique et la qualité de la relation hiérarchique est significative $(\beta=0,11, p<0,001)$. L'hypothèse $H 1$ selon laquelle l'intensité perçue d'utilisation de la messagerie électronique est en relation avec la qualité de la relation hiérarchique est validée. En revanche, lorsque nous introduisons les variables médiatrices, la relation directe n'est plus validée $((\beta=0,09)$.

\section{Effets médiateurs de la reconnaissance, du contrôle et de l'accessibilité}

Les résultats font apparaître deux relations significativement positives : I'intensité perçue d'utilisation de la messagerie électronique sur la reconnaissance $(\beta=0,21, p<0,001)$ et l'intensité perçue d'utilisation de la messagerie sur le contrôle $(\beta=0,24 ; p<0,001)$. En revanche, la relation entre l'intensité perçue d'utilisation de la messagerie et l'accessibilité $n$ 'est pas significative $((\beta=0,05$, $p=0,25$ ).

Afin de tester les hypothèses $\mathrm{H} 2$ et $\mathrm{H}, 3$ selon lesquelles la reconnaissance et le contrôle médiatisent la relation entre l'intensité perçue l'utilisation de la messagerie électronique et la qualité de la relation hiérarchique, nous avons réalisé un test de Sobel (1982). Le test de Sobel vérifie l'effet médiateur d'une première variable sur la relation entre une deuxième et troisième variable. La statistique $z$ de Sobel est significative $(p<0,001)$ pour la médiation des variables reconnaissance et contrôle sur la relation entre l'intensité perçue d'utilisation de la messagerie électronique et la qualité de la relation hiérarchique. Suivant la démarche d'lacobucci et Duhacheck (2003), ces deux effets indirects expliquent respectivement $34,2 \%$ et $36,7 \%$ de l'effet total de l'intensité perçue d'utilisation de la messagerie électronique sur la qualité de la relation hiérarchique. Les hypothèses $\mathrm{H} 2$ et $\mathrm{H} 3$ sont validées.

L'hypothèse H4 selon laquelle l'accessibilité médiatise l'effet de l'utilisation de la messagerie électronique sur la qualité de la relation hiérarchique n'est que partiellement validée dans la mesure où le lien entre utilisation du média et accessibilité n'est pas significatif alors que celui entre accessibilité et LMX I'est.

Le tableau 6 expose la synthèse de la validation des hypothèses. 


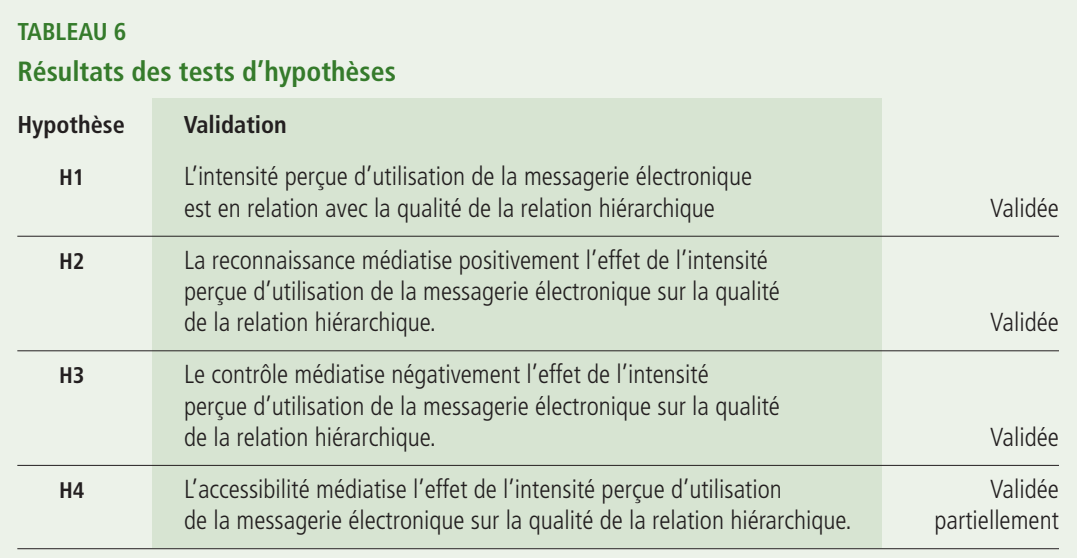

\section{Les divergences entre cadres et non-cadres}

Nous avons utilisé une méthode de comparaison de groupes (PLS-MGA; Haire et al., 2014) pour tester notre modèle sur deux sous-échantillons : les cadres et les non-cadres. II y a une différence significative entre ces deux groupes pour deux relations. La consultation du courrier électronique contribue plus à la perception de l'intensité de l'utilisation de la messagerie électronique pour les cadres que pour les non-cadres ( $\beta$ cadres- $\beta$ non-cadres $=0,13 ; p<0,001$ ), et la relation entre accessibilité et la qualité de la relation hiérarchique est plus forte pour les cadres que pour les non-cadres ( $\beta$ cadres- $\beta$ non-cadres $=0,45 ; p<0,001)$.

\section{Discussion des résultats}

\section{La relation directe entre intensité perçue d'utilisation de la messagerie électronique et qualité de la relation hiérarchique}

Nous constatons tout d'abord que, prise isolément des variables médiatrices, la relation directe entre l'intensité perçue d'utilisation de la messagerie électronique et la qualité de la relation hiérarchique est validée. Ce qui nous amène à conclure que l'intensité perçue par le subordonné lorsqu'il envoie, reçoit des messages ou consulte sa messagerie est liée positivement à la qualité de la relation avec son supérieur hiérarchique. Autrement dit, plus l'intensité perçue d'utilisation du média est forte, plus la qualité de la relation avec le supérieur hiérarchique augmente. Cela renforce la conviction exprimée par Nasr et al. (2009), selon laquelle les interactions entre les acteurs, grâce à un processus d'auto-renforcement, sont censées créer une relation d'échanges de haute qualité. Le collaborateur qui communique intensément avec son supérieur hiérarchique se sent investi dans son service, il se sent au cœur de l'échange social. 
En revanche, lorsque nous traitons le modèle dans son ensemble - en introduisant les variables médiatrices — la relation directe entre utilisation de la messagerie électronique et qualité de la relation hiérarchique n'est plus validée. Autrement dit, lorsque l'on intègre les variables mesurant la façon dont on utilise le média pour exprimer de la reconnaissance, contrôler et se rendre plus accessible, l'intensité perçue d'utilisation n'est plus en relation avec la qualité de la relation hiérarchique. La façon dont on utilise le média prend le pas sur l'intensité perçue avec laquelle on l'utilise; nous rejoignons en cela l'étude de Mano et Mesch (2010), qui concluent que ça n'est pas l'intensité d'utilisation du média qui produit des effets organisationnels, mais la façon dont on I'utilise. Ce ne sont donc pas les qualités intrinsèques de l'outil qui améliorent ou détériorent la relation, mais bien l'usage que l'on en fait. La technologie existe parce qu'on l'évoque, on la paramètre, on l'utilise (de Vaujany, 2009) : dans le cas présent, la messagerie existe et elle est en relation avec l'échange social parce qu'on l'utilise pour reconnaître le travail du salarié, le contrôler, ou encore accroître l'accessibilité.

Notons, enfin, que dans la mesure de l'intensité perçue de l'utilisation du média, la place que tient la consultation du média prend plus d'importance pour les cadres que pour les non-cadres. Une consultation répétée de sa boîte de courrier électronique par l'utilisateur est un signe de sa dépendance au média (de la Rupelle, 2010); et nos résultats permettent de conclure que ce phénomène est plus visible chez les cadres que chez les non-cadres.

\section{La médiation de la reconnaissance, du contrôle et de l'accessibilité}

Les résultats de l'étude du modèle par équations structurelles montrent que la reconnaissance joue un rôle médiateur entre l'utilisation du média et la qualité de la relation hiérarchique. L'intensité perçue d'utilisation du média est liée positivement à la reconnaissance qui, à son tour, est liée positivement à la qualité de la relation hiérarchique. Cette conclusion est majeure dans la mesure où elle indique aux managers l'importance de la prise en compte du média dans les mécanismes de reconnaissance : remercier, féliciter, encourager par messagerie, transmettre de l'information à ses collaborateurs et les rendre ainsi plus autonomes, ou répondre rapidement à un message sont autant d'actions qui sont liées positivement à la qualité de l'échange social avec les subordonnés. Le mécanisme de la reconnaissance s'imbrique parfaitement dans le cadre de l'échange social dans la mesure où elle est un processus de communication mutuel qui émerge dans l'interaction; la reconnaissance n'est pas un simple échange, c'est un partage (Andonova et Vacher, 2009), on parle même d'exigence de réciprocité.

Les résultats concernant le rôle médiateur du contrôle sont tout aussi porteurs de sens : I'intensité perçue d'utilisation du média est liée positivement au contrôle, mais celui-ci est lié négativement à la qualité de la relation hiérarchique. 
La messagerie électronique permet de conserver des traces écrites de l'activité d'un collaborateur, de contrôler l'état d'avancement d'une tâche, de s'assurer du respect des procédures ou encore de formaliser des points abordés à l'oral. Le média, considéré a priori comme étant inoffensif, devient alors un puissant dispositif de contrôle et de surveillance. Le contrôle sous toutes ses formes énoncées précédemment est lié négativement avec la qualité de la relation hiérarchique. Plus le collaborateur se sent sous surveillance, plus la qualité de la relation avec son supérieur en pâtit. Cette démonstration peut s'enrichir des conclusions de Rosanvallon (2011), qui affirme que cette perception de surveillance « se construit bien sur des expériences réelles de ce contrôle, mais elle se nourrit aussi du doute qui plane inévitablement autour des usages réels que l'encadrement fait des outils » (21). Il est fondamental que les managers réalisent que trop de contrôle par le biais du média peut conduire à dégrader la qualité de la relation hiérarchique : les salariés vont, dès lors, avoir tendance à se protéger et à limiter leurs échanges (Rosanvallon, 2011).

Nous analysons, enfin, l'effet médiateur de l'accessibilité et constatons que I'hypothèse de la relation entre l'utilisation de la messagerie électronique et I'accessibilité n'est pas validée. En revanche, I'amélioration de sa propre accessibilité ou de celle de son supérieur hiérarchique en cas d'absence est liée positivement à la qualité de la relation hiérarchique ; parce qu'elle permet de garder le lien, la messagerie électronique va dans le sens de la qualité de la relation entre supérieurs hiérarchiques et subordonnés. Mieux gérer sa propre absence, s'affranchir des contraintes spatiales, rester informé de l'actualité de l'entreprise ou de son service en cas d'absence sont autant d'atouts qui vont dans le sens d'une meilleure relation sociale. Cette relation entre accessibilité et qualité de la relation hiérarchique est encore plus forte pour les cadres que les non-cadres; cette amplification du phénomène s'explique très certainement par les déplacements plus nombreux et plus fréquents pour les cadres que pour les non-cadres et la possibilité que procure alors le média pour rester connecté.

\section{Implications théoriques et pratiques}

D'un point de vue théorique, l'étude présentée offre plusieurs contributions à la recherche. Elle propose, tout d'abord, un modèle construit et articulé sur des bases théoriques permettant de mieux expliquer la relation entre l'intensité perçue de l'utilisation de la messagerie électronique et la qualité de la relation hiérarchique, en introduisant des variables intermédiaires telles que la reconnaissance, le contrôle ou encore l'accessibilité.

Nous sommes, par ailleurs, en mesure de proposer de nouveaux instruments de mesure. L'échelle à trois construits, élaborée pour mesurer l'intensité perçue d'utilisation du média, permet de tester la perception de l'utilisateur alors que les 
études cherchent habituellement à en avoir une mesure métrique. Cet instrument de mesure permet, dès lors, d'étudier le phénomène à travers le prisme de ce que ressent I'utilisateur et non par le biais d'un volume objectif dénué de toute appréciation personnelle. Les trois échelles construites pour tester nos variables médiatrices sont également des instruments de mesure inédits dans la recherche. Elles autorisent la prise en compte du poids du média dans les mécanismes qu'elles mesurent entre un collaborateur et son supérieur hiérarchique.

D'un point de vue pratique, la première conclusion fondamentale à retenir réside dans le fait que, prise indépendamment des variables médiatrices, l'intensité perçue d'utilisation du média présente une relation positive avec la qualité de la relation hiérarchique. Cela signifie qu'entretenir le lien avec ses subordonnés, par le biais de la communication électronique, de façon soutenue est plutôt porteur de sens et va dans le sens d'une bonne qualité de la relation. Le collaborateur y voit un intérêt de son manager, une marque de confiance ou a l'impression d'être au cœur de l'action. Mais cette première piste, du point de vue des pratiques de gestion, n'est que parcellaire puisque lorsque nous introduisons les variables médiatrices que sont la reconnaissance, le contrôle et l'accessibilité, l'intensité perçue du média n'est plus liée avec la qualité de la relation hiérarchique. Ce ne sont donc pas les qualités intrinsèques de l'outil qui améliorent ou détériorent la relation, mais plutôt l'usage que l'on en fait. La technologie existe parce qu'on l'évoque, on la paramètre, on l'utilise (de Vaujany, 2009) : la messagerie existe parce qu'on l'utilise pour reconnaître le travail du salarié, le contrôler, ou encore accroître l'accessibilité..., et ce sont ces usages qui produisent des effets sur la dyade supérieur hiérarchique/subordonné. Notre étude démontre qu'utiliser la messagerie pour des marques de reconnaissance ou se rendre plus accessible produit des effets positifs sur la qualité de la relation hiérarchique et qu'au contraire, utiliser la messagerie pour contrôler, produit des effets négatifs. Ces résultats peuvent conduire les managers à une prise de conscience des conséquences de ces résultats, puis à la mise en place d'interventions visant à améliorer les pratiques de communication électronique. Un supérieur hiérarchique pressentant une dégradation de la qualité de la relation avec un subordonné pourra ainsi activer les mécanismes de reconnaissance par le biais du média. La reconnaissance doit s'exprimer « de manière visible, évidente, palpable, matérielle pour que l'individu puisse la saisir » (Andonova et Vacher, 2009). La messagerie électronique constitue un moyen pour le supérieur hiérarchique de formaliser sa reconnaissance à l'égard d'un subordonné. Dans le prolongement des travaux de St-Onge et al. (2005), nous mettons cependant le lecteur en garde contre une utilisation abusive, manipulatrice ou non alignée sur les priorités. Octroyer trop de reconnaissance peut être une forme de manipulation puisque le système pousse les subordonnés " à faire juste ce qu'il faut pour les obtenir », à ne s'engager que sur les activités rentables en terme de reconnaissance et à délaisser les autres. 
La reconnaissance et le contrôle pourront être intégrés dans le cadre de la communication avec le collaborateur à titre de leviers d'action et d'ajustement de la qualité de l'échange. Nous formons le vœu que notre étude conduise les managers à remettre en cause, à nuancer et à éclairer leurs pratiques dans les échanges électroniques. Tout en restant conscients du fait que la communication « virtuelle » n'est que le prolongement de la communication « réelle », ils devraient être aptes à en optimiser l'utilisation dans un processus de régulation en vue d'améliorer la qualité de la relation hiérarchique (Nasr et al., 2009).

\section{Conclusion}

L'objectif de cette étude était de vérifier la relation entre l'intensité perçue d'utilisation de la messagerie électronique et la qualité de la relation hiérarchique, puis de vérifier la médiation de variables telles que la reconnaissance, le contrôle ou encore l'accessibilité. Les résultats nous permettent d'affirmer que la relation positive entre intensité perçue d'utilisation du média et qualité de la relation hiérarchique, mais que cette relation disparaît dès lors que l'on introduit les variables médiatrices de reconnaissance, contrôle et accessibilité. Les marques de reconnaissance exprimées à travers la messagerie électronique, ainsi que l'accessibilité accrue qu'elle permet, améliorent la qualité de la relation hiérarchique, tandis que les actions de contrôle la détériorent.

Les résultats de notre étude comportent des limites, et, parmi elles, le fait que les répondants soient en grande partie des cadres (74\% des personnes interrogées). Si la constitution de l'échantillon de départ était bien représentative sur ce critère, il s'avère que ce sont les cadres qui ont très majoritairement répondu à l'enquête. Nous pouvons avancer deux explications au phénomène : les cadres sont sollicités par messagerie, peut-être plus encore que les non-cadres, et se sentent donc plus concernés, voire préoccupés par l'utilisation du média. II se peut par ailleurs que les non-cadres aient eu plus de réticences à répondre à un questionnaire incisif sur la relation hiérarchique.

D'un point de vue méthodologique, si l'analyse par équations structurelles permet d'étudier simultanément de multiples relations entre un ensemble de variables, elle rencontre aussi des limites. Parmi elles, notons la difficulté d'employer l'analyse par équations structurelles lorsqu'il existe des relations circulaires entre les variables. Ainsi, dans le cadre de notre étude, nous n'avons pas testé l'existence d'une boucle de causalité entre la qualité de la relation hiérarchique et l'intensité perçue de l'utilisation de la messagerie électronique. Ceci pourrait faire l'objet d'un projet de recherche futur.

En outre, nos mesures sont basées sur des perceptions: nous avons choisi volontairement de mesurer I'utilisation de la messagerie électronique par la per- 
ception qu'en ont les subordonnés et non par des mesures métriques du nombre de messages reçus ou envoyés quotidiennement, par exemple. Cela nous permet d'étudier le phénomène sous l'angle de ce que ressent l'utilisateur : il serait néanmoins profitable, à l'occasion d'une étude à venir, de comparer la perception qu'a l'utilisateur de sa messagerie électronique à la volumétrie réelle de ses envois, réceptions et consultations.

De la même façon, d'autres variables de contingence jouant un rôle modérateur pourront être examinées, telles la distance géographique, la personnalité du répondant ou encore le contexte organisationnel et/ou technique.

Enfin, l'étude se concentre exclusivement sur la perception des personnes interrogées dans leur position de subordonné. II serait intéressant de mettre en œuvre une étude miroir sur la perception des supérieurs hiérarchiques afin d'aller plus en avant sur la réciprocité de la relation si chère à la théorie de l'échange social.

\section{Bibliographie}

Alie, Karine. 2007. "Comparaison de deux outils mesurant certains aspects de la mobilisation du personnel dans une organisation de soins de santé et de services sociaux ». Thèse de doctorat soutenue en décembre 2007. Université de Sherbrooke.

Andonova, Yanita et Béatrice Vacher. 2009. "Visibilité et reconnaissance de I'individu au travail ». Communication et Organisation, 36, 136-147.

Assadi, Houssem. et Jérôme Denis. 2005. "Les usages de l'e-mail en entreprise : efficacité dans le travail ou surcharge informationnelle? » in Kessous Emmanuel et Jean-Luc Metzger. Le travail avec les technologies de l'information. Éditions Lavoisier, Collection Technique et Scientifique des Télécommunications, 136-155.

Ayache, Magali et Hervé Laroche. 2010. "La construction de la relation managériale ». Revue Française de Gestion, 36 (203), 133-147.

Bachelet, Catherine et Jean Moscarola. 2001. "La messagerie électronique, facteur de changement dans I'organisation ? Implication sur la décision ». $5^{\text {ème }}$ colloque du CR/C.

Bia Figueiredo, Maria et Michel Kalika. 2010. La communication électronique. Economica.

Biocca, Frank et Chad Harms. 2002. « Defining and measuring social presence: contribution to the networked minds theory and measure ». Proceedings of the 5th International Workshop on Presence, 1-36.

Blau, Peter Michael. 1964. Exchange and Power in Social Life. New York: Wiley.

Boukef, Nabila. 2005. "Utilisation du courrier électronique dans l'activité managériale : usages, intérêt et limites». Thèse de doctorat, soutenue le 06 décembre 2005. Université Paris Dauphine.

Boukef-Charki, Nabila et Mohamed Hedi Charki. 2009. "L'e-mail : un moyen de contrôle ou de responsabilisation ? ». Systèmes d'Information et Management, 13 (4), 31-60. 
Cerdin, Jean-Luc et Jean-Marie Peretti. 2001. "Internet versus voie postale : comparaison de deux méthodes de collecte de données en GRH ». Revue de gestion des ressources humaines, 42, 39-56.

Churchill, Gilbert A. 1979. " A Paradigm for Developing Better Measures of Marketing Constructs ». Journal of Marketing Research, 16 (1), 64-73.

Craipeau Sylvie et Bertrand Seys. 2005. "Jeux et Internet : quelques enjeux psychologiques et sociaux ». Psychotropes, 11, 101-127.

Creswell, John W. 2003. Research Design - Qualitative, Quantitative, and Mixed Methods Approaches. Sage Publications

Duchenneaut, Nicolas et Victoria Bellotti. 2001. «Email as Habitat - An Exploration of Embedded Personal Information Management ». Interactions, 8 (5), 30-38.

Daft, Richard L. et Robert H. Lengel. 1986. « Organization Information Requirements, Media Richness and Structural Design ». Management Science, 32 (5), 554-571.

Dansereau, Fred, George Graen et William J. Haga. 1975. «A Vertical Dyad Linkage Approach to Leadership within Formal Organizations: A Longitudinal Investigation of the Role Making Process ». Organizational Behavior and Human Performance, 13 (1), 46-78.

El Akremi Assaad, Narjes Sassi et Sihem Bouzidi. 2009. "Rôle de la reconnaissance dans la construction de l'identité au travail ». Relations industrielles/Industrial Relations, 64 (4), 662-684.

Forgues, Bernard. 1995. "La traduction des questionnaires ». AFM Nouvelles du Marketing, 34, 1-19.

Fornell, Claes et David F. Larcker. 1981. "Structural Equation Models with Unobservable Variables and Measurement Errors ». Journal of Marketing Research, 18 (1), 39-50.

Foucault, Michel. 1975. Surveiller et punir, naissance de la prison. 1ère édition, 1975, Paris Gallimard, 360 pages.

Giddens, Anthony. 1989. "A Reply to my Critics », dans Social Theory of Modern Societies: Anthony Giddens and His Critics, Held D and Thompson JB (Eds), Cambridge University Press, Cambridge.

Goh, Samuel et Molly Wasko. 2012. "The Effects of Leader-member Exchange on Member Performance in Virtual World Teams ». Journal of the Association for Information Systems, 13 (10), 861-885.

Graen, George et James Cashman. 1975. "A Role-making Model of Leadership in Formal Organizations: a Development Approach », dans J.G. Hunt et L.L. Lawson (Eds), « Leadership frontiers ». Kent State University Press, 143-165.

Graen, George, Mike Novak et Pat Sommerkamp. 1982. "The Effects of Leader-member Exchange and Job Design on Productivity and Satisfaction: Testing a Dual Attachment Model ». Organization Behavior and Human Performance, 30, 109-131.

Graen, George et Mary Uhl-Bien. 1995. " Relationship-based Approach to Leadership: Development of Leader-Member Exchange (LMX) Theory of Leadership over 25 years: Development of Level Multi-domain Perspective ». Leadership Quarterly, 6 (2), 219-247.

Greenan, Nathalie, Sylvie Hamon-Cholet, Frédéric Moatty et Jérémie Rosanvallon. 2012 . «TIC et conditions au travail, les enseignements de l'enquête COI ». Rapport de recherche Centre d'Études et d'Emploi, 72 pages. 
Griffith, Terry, John E. Sawyer et Margaret A. Neal. 2003. "Virtualness and Knowledge in Teams: Managing the Love Triangle of Organizations, Individuals, and Information technology ». MIS Quarterly, 27 (2), 265-287.

Hair Jr, Joseph F., G Tomas M Hult, Christian M Ringle et Marko Sarstedt. 2013. A Primer on Partial Least Squares Structural Equation Modeling (PLS-SEM). SAGE Publications, Incorporated.

Hinds, Pamela et Diane E. Bailey. 2003. "Out of Sight, Out of Sync.: Understanding Conflict in Distributed Teams ». Organization Science, 14 (6), 615-632.

Hildreth, Andrew K.G. 2001. «A New Voice or a Waste of Time? Wage Premiums from Using Computers for Communication in the UK Workplace ». British Journal of Industrial Relations, 39 (2), 257-284.

Hinkin, Timothy R. 2005. "Scale Development Principles and Practices ». In Richard A. Swanson et Elwood F. Holton (Eds.), Research in Organizations: Foundations and Methods of Inquiry (1st ed.). San Francisco: Berrett-Koehler Publishers Inc., 161-179.

Hodson, Randy. 2001. Dignity at Work. Cambridge University Press. 320 pages.

Howell, Jane M., Derrick J. Neufeld et Bruce Avolio. 2005. "Examining the Relationship of Leadership and Physical Distance with Business Unit Performance ». Leadership Quarterly, $16,273-285$.

Huang, Albert H. 2002. " E-mail Communication and Supervisor-subordinate Exchange Quality: an Empirical Study ». Human Systems Management, 21 (3), 193-204.

lacobucci, Dawn et Adam Duhachek. 2003. " Mediation Analysis: Round table ACR 2003 ». Présentation à la table ronde de l'ACR 2003, Toronto.

Kalika, Michel, Nabila Boukef et Henri Isaac. 2007 « La théorie du millefeuille et I'usage des TIC dans I'entreprise ». Revue française de gestion, 33 (172), 117-133.

Klein, Howard J. et Jay S. Kim. 1998. "A Field Study of the Influence of Situational Constraints, Leader-member Exchange, and Goal Commitment on Performance ». Academy of Management Journal, 41 (1), 88-95.

Lalle, Béatrice. 1999. « Nouvelles technologies et évolution de la dialectique (contrôle/autonomie) dans le secteur des services application au cas bancaire ». Revue de gestion des ressources humaines, 32, 97-107.

La Rupelle (de), Géraldine et Michel Kalika. 2009. "Messagerie électronique et relations hiérarchiques : union parfaite ou impossible mariage ? ", Management et Avenir, 30, $51-74$.

La Rupelle (de), Géraldine. 2010. «Les effets de I'utilisation de la messagerie électronique sur la relation hiérarchique ». Thèse de doctorat soutenue le 16 novembre 2010, Université Paris Dauphine.

Leclercq, Aurélie. 2008. « Le contrôle organisationnel et les technologies et systèmes d'information mobiles : une approche foucaldienne ». Thèse de doctorat soutenue le 14 mai 2008. Université Paris Dauphine.

Lemarie, Yves. et Line Wagemann. 2000. " Analyse des situations de communication par messagerie électronique chez les cadres dirigeants dans leur rôle de leader ». Systèmes d'information et management, 5 (3), 137-153.

Linden, Robert et George Graen. 1980. « Generalizability of the Vertical Dyad Linkage Model of Leadership ». Academy of Management Journal. 23 (3), 451-465. 
Klein, Howard J. et Jay S. Kim. 1998 « A Field Study of the Influence of Situational Constraints, Leader-member Exchange, and Goal Commitment on Performance ». Academy of Management Journal, 41 (1), 88-95.

Malhotra, Naresh K., Jean-Marc Decaudin et Afifa Bouguerra. 2004. Études marketing avec SPSS. Pearson Éducation France, 664 pages.

Mano, Rita et Gustavo S. Mesch G.S. 2010. "E-mail Characteristics, Work Performance and Distress », Computers in Human Behavior, 26, 61-69.

Mc Manus, Denis, Chetan Sankar, Houston H. Carr et F. Nelson Ford. 2002. « Intra-organizational versus Inter-organizational Uses and Benefits of Electronic Mail ». Information Ressources Management Journal, 15 (3), 1-13.

Morin, Fernand. 2000. "Nouvelles technologies et la télésubordination du salarié ». Relations industrielles/Industrial Relations, 55 (4), 725-745.

Nasr, Mohamed Ikram, Assâad El Akremi et Christian Vandenberghe. 2009. " Justice organisationnelle, confiance et comportements de citoyenneté : test d'un modèle multicibles de l'échange social au travail ». Revue de gestion des ressources humaines, 74, 3-23.

Niedle, Larisa. 2012. "A Comparison of LMX, Communication, and Demographic Differences in Remote and Co-located Supervisor-Subordinate Dyads », College of Science and Health Theses and Dissertations, Paper 17.

Nolan, Dennis R. 2003. "Privacy and Profitability in the Technological Workplace ». Journal of Labor Research, 24 (2), 207-232.

Nunnally, Jum C. 1978. Psychometric Theory. New York: McGraw-Hill.

Orlikowski, Wanda.J. 1992. "The Duality of Technology: Rethinking the Concept of Technology in Organization ». Organization Science, 3 (3), 398-427.

Ringle, Christian M., Sven Wende et Alexander Will. 2005. SmartPLS Version 2.0 (beta). Hamburg, Germany: SmartPLS.

Romelaer, Pierre. 2005. «L'entretien de recherche » dans Roussel P. et Wacheux F., Management des ressources humaines, méthodes de recherche en sciences humaines et sociales, de Boeck Université, 101-138.

Rosanvallon, Jérémie. 2011. "Le contrôle du travail, entre réalités et perceptions : le cas de la messagerie électronique ». Sociologie pratique, 1 (22), 19-33.

Saint-Michel, Sarah et Nouchka Wielhorski. 2011. "Style de leadership, LMX et engagement organisationnel des salariés : le genre du leader a-t-il un impact ? ». @GRH, 1, 13-38.

Simard, Gilles, Olivier Doucet et Sarah Bernard. 2005. «Pratiques en GRH et engagement des employés, le rôle de la justice ». Relations industrielles/Industrial Relations, 60 (2), 296-319.

Sobel, Michael E. 1982. "Asymptotic Confidence Intervals for Indirect Effects in Structural Equation Models ». Sociological Methodology, 13, 290-312.

Sproull, Lee et Sara Kiesler. 1991. Connections: New Ways of Working in the Networked Organization. Cambridge, MA: MIT Press, 205 pages.

St-Onge, Sylvie, Haines Victor Y. III, Isabelle Aubin, Claudia Rousseau et Geneviève Lagassa. 2005. «Pour une meilleure reconnaissance des contributions au travail ». Gestion, 30 (2), 89-101.

Straus, Susan G. et Joseph E. Mc Grath. 1994. "Does the Medium Matter? The Interaction of Task Type and Technology on Group Performance and Member Reactions ». Journal of Applied Psychology, 79 (1), 87-97. 
Tahri, Wadi et Bernard Fallery B. 2009. " La messagerie électronique : une technologie devenue banale mais toujours en évolution ". Réseaux numériques et nouvelles frontières Organisationnelles, Amérique du Nord, nov. 2009.

Tahri, Wadi et Bernard Fallery B. 2010. "L'usage de la messagerie électronique : une métaanalyse des travaux francophones sur la période 2000-2008». Management et Avenir, 4 (34), 184-200.

Taylor, Howard, George Fieldman et Yochanan Altman. 2008. "E-mail at Work: a Cause for Concern? The Implications of the New Communication Technologies for Health, Wellbeing and Productivity at Work ». Journal of Organizational Transformation and Social Change, 5 (2), 159-172.

Thietart, Raymond-Alain et collaborateurs. 2007. Méthodes de recherche en management. Dunod, Paris, 586 pages.

Van den Hooff, Bart. 2005. " A Learning Process in E-mail Use - a Longitudinal Case Study of the Interaction between Organization and Technology ». Behaviour and Information Technology, 24 (2), 131-145.

Vaujany (de), François-Xavier. 2001. "Gérer I'innovation sociale à l'usage des technologies de I'information : une contribution structurationniste », Thèse de doctorat, Université Jean Moulin Lyon 3.

Vaujany (de), François-Xavier. 2009. Les grandes approches théoriques du système d'information, Hermès Lavoisier, 239 pages.

Wallace, Jean E. 2005. « Job Stress, Depression and Work-to-Family Conflict : a Test of the Strain and Buffer Hypotheses », Relations industrielles/Industrial Relations, 60 (3), 510-537.

Wils, Thierry et Christiane Labelle. 2005. "Faut-il se soucier de ses professionnels pour les mobiliser ? », Revue Gestion, 30 (2), 37-49.

\section{RÉSUMÉ}

\section{La relation entre l'intensité perçue d'utilisation de la messagerie électronique et la qualité de la relation hiérarchique}

Stress, conflits, surcharge, pression et contrôle, mais aussi gain de temps, motivation, accessibilité... sont autant de mots souvent prononcés à l'évocation de la messagerie électronique, tout particulièrement lorsqu'elle est associée aux relations hiérarchiques.

Bien que les chercheurs et praticiens reconnaissent les effets du média sur la relation hiérarchique, la recherche académique peine à expliquer la nature de leur relation ainsi que les variables explicatives de cette même relation. Visant à répondre à ces questions, la problématique centrale de notre étude consiste à examiner la relation entre l'utilisation de la messagerie électronique et la qualité de la relation hiérarchique en mettant l'accent sur trois variables médiatrices : la reconnaissance, le contrôle et l'accessibilité. 
L'étude empirique a été réalisée au sein d'une banque de réseau française. Une étude qualitative exploratoire a contribué à la construction d'échelles de mesure utilisées ensuite dans un dispositif de recherche quantitatif : 478 questionnaires valides ont permis une analyse du modèle par la méthode des équations structurelles.

Les résultats révèlent que l'intensité perçue d'utilisation du média est liée positivement à la qualité de la relation hiérarchique lorsque la relation est considérée isolément des variables médiatrices. En revanche, lorsque nous traitons le modèle dans son ensemble - en introduisant les variables médiatrices de reconnaissance, contrôle et accessibilité - la relation directe entre utilisation de la messagerie électronique et qualité de la relation hiérarchique n'est plus validée. La reconnaissance et le contrôle sont des mécanismes explicatifs de cette relation, tandis que I'accessibilité n'explique que partiellement la relation entre messagerie électronique et qualité de la relation hiérarchique. Outre les apports méthodologiques que procure la construction du modèle et celle des échelles de mesure, la présente recherche vise à fournir des pistes pour une amélioration des pratiques de communication électronique au sein de la dyade supérieur hiérarchique/subordonné.

MOTS-CLÉS : communication électronique, courrier électronique, relation managériale, théorie de l'échange social, relation leader-membre (LMX en anglais).

\section{SUMMARY}

\section{The relationship between perceived intensity of email use and quality of hierarchical relations}

Stress, disputes, work overload, pressure, and control but also time saving, acknowledgment, accessibility.... are all words that are frequently used when talking about e-mail, and particularly in connection with hierarchical relations.

While researchers and practitioners do acknowledge the effects that this tyoe of media has on hierarchical relations, they struggle to explain the nature of the causal relationships at work. The aim of this paper is to answer these questions. We examine the relationship between the use of e-mail and the quality of hierarchical relations by highlighting three central mediating variables: acknowledgment, control and accessibility.

Data was collected on e-mail use by 478 employees in a French retail bank. We used structural equation modeling to test our hypotheses. The results reveal that the perceived intensity of e-mail use has a direct positive effect on the quality of hierarchical relations when tested independently of mediating variables. However, this direct effect is not significant when the mediating variables of acknowledgment, control and accessibility are introduced into the model. Our study contributes to our understanding of the impact of electronic communication on the dyadic relationship between a superior and his or her subordinates.

KEYWORDS: Electronic communication, e-mail, managerial relation, social exchange theory, leader-member exchange theory (LMX). 


\section{RESUMEN}

\section{La relación entre la intensidad percibida de utilización de la mensajería electrónica y la calidad de la relación jerárquica}

Estrés, conflictos, sobrecarga, presión y control, pero también economía de tiempo, motivación, accesibilidad... son tantas palabras pronunciadas a la evocación de la mensajería electrónica, particularmente cuando ésta es asociada a las relaciones jerárquicas.

Aunque los investigadores y los profesionales reconocen los efectos del medio sobre la relación jerárquica, la investigación académica tarda a explicar la naturaleza de dicha relación y de las variables explicativas de esta misma relación. Buscando respuestas a estas preguntas, la problemática central de nuestro estudio consiste en examinar la relación entre la utilización d la mensajería electrónica y la calidad de la relación jerárquica poniendo el acento en tres variables mediadoras: el reconocimiento, el control y la accesibilidad.

El estudio empírico fue realizado en un banco de red en Francia. Un estudio cualitativo exploratorio ha contribuido a la construcción de escalas de medida utilizadas enseguida en un dispositivo de investigación cuantitativa: 478 cuestionarios validos permitieron un análisis del modelo mediante el método de ecuaciones estructurales.

Los resultados revelan que la intensidad percibida de utilización del medio está asociada positivamente a la calidad de la relación jerárquica cuando la relación es considerada de manera aislada de las variables mediadoras. Al contrario, cuando se trata el modelo en su conjunto - introduciendo las variables mediadoras de reconocimiento, control y accesibilidad - la relación directa entre utilización de la mensajería electrónica y calidad de la relación jerárquica no es significativa. El reconocimiento y el control son los mecanismos explicativos de esta relación mientras que la accesibilidad explica solo parcialmente la relación entre mensajería electrónica y calidad de la relación jerárquica. Más allá de los aportes metodológicos que constituyen la construcción del modelo y el diseño de las escalas de medida, la presente investigación intenta ofrecer pistas para mejorar las prácticas de comunicación electrónica en medio del dúo superior jerárquico/subordinado.

PALABRAS CLAVES: comunicación electrónica, correo electrónico, relación de gestión, teoría del intercambio social, relación líder-miembro (LMX). 